\title{
LA RECONQUISTA URBANA DEL ESPACIO DE PROXIMIDAD. LOS PATIOS INTERIORES EN EL EIXAMPLE DE BARCELONA
}

Teresa PAZOS ORTEGA

En este artículo se exponen algunas conclusiones del estudio de la funcionalidad de los patios interiores públicos del Eixample de Barcelona, nueva tipología de espacio libre. Se llevó a cabo la observación directa del uso de ciertos patios concretos que sirvieron como modelo para comparar la variedad de comportamientos según los distintos tipos seleccionados, funcionalidad del espacio, intensidad de uso, actividades desarrolladas, relación entre sí y con otros elementos urbanos y tipo de usuarios. La información se complementó con encuestas de opinión sobre hábitos de uso y preferencias. Los resultados aportan información útil a la hora de crear nuevos espacios y mantener, con actividad, los que ya existen. Confirman la funcionalidad de los patios y su gran utilidad dentro de la trama urbana. El esfuerzo llevado a cabo por el ayuntamiento tanto económico como en gestión merece la pena y debe seguir haciéndose: los beneficios son mayores a las cargas.

Funcionalidad del espacio público, patios interiores, uso de proximidad

\section{THE URBAN RECONQUEST OF THE SPACE OF PROXIMITY. OPEN INTERIOR COURTYARDS AT BARCELONA'S EIXAMPLE}

This paper applies an approach to the use and adaptability of the courtyards or interior open spaces (IOS) of Barcelona's Eixample city blocks. A number of city block IOS were selected as models to asses, via direct observation, their functionality within the general system of open spaces. The objective was to evaluate the functionality of the spaces, intensity of use, developed activities, relationship with other IOS and external urban elements, as well as users' profiles and behaviours. Direct observation was completed with qualitative surveys to find out attitudes, purposes and preferences of regular users. The results provide useful information for the design of new IOS and the maintenance of current ones. They also confirm that the efforts made by the City Council both economically and management are worthwhile and should continue to be made: the benefits outweigh the burdens.

Adaptability of public spaces, interior open spaces, vicinity uses
Los espacios públicos son costosos de obtener y de mantener, especialmente en ciudades densas como en Barcelona y en concreto en su Eixample, que se encuentra altamente densificado. Los patios interiores de manzana y otras zonas ajardinadas del mismo son oasis dentro de la trama, hechos excepcionales fruto del esfuerzo del ayuntamiento y entidades privadas. Es necesario comprobar su utilización y funcionalidad para verificar que los esfuerzos económicos y de gestión son una buena inversión. La buena opinión de los ciudadanos, la seguridad o el uso son indicadores de un buen estado de los espacios, del barrio y de la ciudad ${ }^{1}$.

En este artículo se exponen algunas conclusiones obtenidas del trabajo de campo realizado en los patios interiores de uso público recuperados en el Eixample. Forma parte de una tesis doctoral más amplia sobre el proceso seguido y sus resultados en el que se considera al patio interior público como una nueva tipología de espacio libre en Barcelona. Este patio posee unas características concretas y unos usos también concretos, complementarios la actividad desarrollada en los equipamientos algunos de los cuales son resultado del mismo proceso de recuperación de los patios interiores de manzana.

\section{Una nueva tipología de espacios libres en el interior de los patios de manzana}

Obtener un espacio libre en una ciudad consolidada no es tarea fácil. Es complejo de gestionar y tiene obviamente un coste altísimo. Con carácter puntual es posible realizar una adquisición o expropiación, pero su generalización a toda la ciudad consolidada es inviable. En general la mejor manera de obtenerlos es la cesión. Posteriormente queda la urbanización, el ajardinamiento y su mantenimiento. Por ello es necesario valorar el enorme y riguroso esfuerzo llevado a cabo por el Ayuntamiento de Barcelona para conseguir, en menos de 25 años hasta 46 piezas de espacio libre en interiores de manzana, solo en el Eixample. Es una iniciativa, sin precedentes por el gran número de casos, para descongestionar y mejorar la calidad urbana y corregir la falta de zonas verdes y equipamientos. La superficie recuperada asciende a 9 ha, una alta cifra teniendo en cuenta la elevada densidad de ocupación de la trama urbana. Aunque lo más interesante de resaltar no es tanto la superficie recuperada como el número de espacios obtenidos. Son peqiueños pero al estar distribuidos por toda la trama son accesibles a más gente (Fig. 1).

1 Este artículo se ha extraído de la tesina del máster de investigación en urbanismo de la ETSAB presentado en febrero de 2012 por la misma autora y dirigida por Miquel Corominas: "Los espacios recobrados para uso público en los patios interiores de manzana del Eixample". 
El esfuerzo ha merecido la pena y como resultado se han ido estableciendo paulatinamente una amplia variedad de espacios libres interiores que han esponjado la densa trama y facilitado unas dotaciones de proximidad. En esta línea innovadora, una vez abiertos al público y al cabo de cierto tiempo, debería realizarse un estudio sobre su correcto papel como pieza urbana, sobre su funcionamiento y uso, de modo que sirva para hacer más eficiente su servicio, averiguar los motivos por los que no ha funcionado y replantear el espacio, buscar soluciones y actuar si fuera necesario; sirven de referencia para los que quedan por hacer.

Los espacios libres deben cumplir, principalmente, tres funciones básicas: sociales, ambientales y culturales. Son lugares de relación y encuentro, de convivencia. El arbolado, la vegetación o el uso de materiales adecuados permiten crear un lugar fresco, protegido y tranquilo. Pero son también un espacio identitario a escala vecinal, donde poder apropiarse de la ciudad y del barrio. Son una inversión a medio y largo plazo para conseguir una mayor cohesión social y una mejor calidad de vida de la ciudad y de sus ciudadanos.

Por las peculiaridades derivadas de su origen y de su propósito de servir a las comunidades locales estos patios interiores de manzana de uso público constituyen una nueva tipología de espacio libre en la ciudad de Barcelona. Se crean en el Eixample siguiendo una estrategia con instrumentos específicos de recuperación de esta zona emblemática de la ciudad, carente de zonas verdes y equipamientos y en un grave proceso de terciarización y degradación. Son recintos cerrados, de uso público pero limitado al horario diurno, con acceso desde la calle por pasillos bajo edificación o pasos abiertos estrechos. Provienen de espacios privados recuperados o de parcelas de gran tamaño ocupadas por naves industriales o almacenes que han sido demolidos y transformados. Su tamaño es inferior al $40 \%$ de la manzana, con una superficie que oscila entre los $400 \mathrm{y}$ los $4.000 \mathrm{~m}^{2}$.

De los 46 espacios recuperados 34 son patios y, de éstos, 15 tienen algún tipo de equipamiento en relación: dotación y zona verde comparten el mismo acceso; funcionan como conjunto, se complementan. Se trata de equipamientos de escala local, como bibliotecas, centros de día de mayores, guarderías, que se adaptan bien a superficies reducidas. Incluso a veces se combinan además varios equipamientos entre $s^{i}{ }^{2}$, aumentando la complejidad del conjunto con la mixticidad de usos. En la mayoría de casos el equipamiento queda en fachada, cerrando el recinto interior y haciendo de puerta, pero en otros casos permanece en interior, siendo el espacio verde su acceso y "vestíbulo". En estos casos de combinación el beneficio mutuo es enorme. Es la situación

2 Uno de los úttimos equipamientos inaugurados en combinación con patio es el de Teresa Pàmies, que incluye biblioteca, guardería y centro cívico. Para el interior de manzana el equipamiento más compatible parece ser la guardería juegos del patio como prolongación de sus instalaciones. Hay hasta cinco guarderías, una de ellas privada, en patios interiores de manzana de uso público.

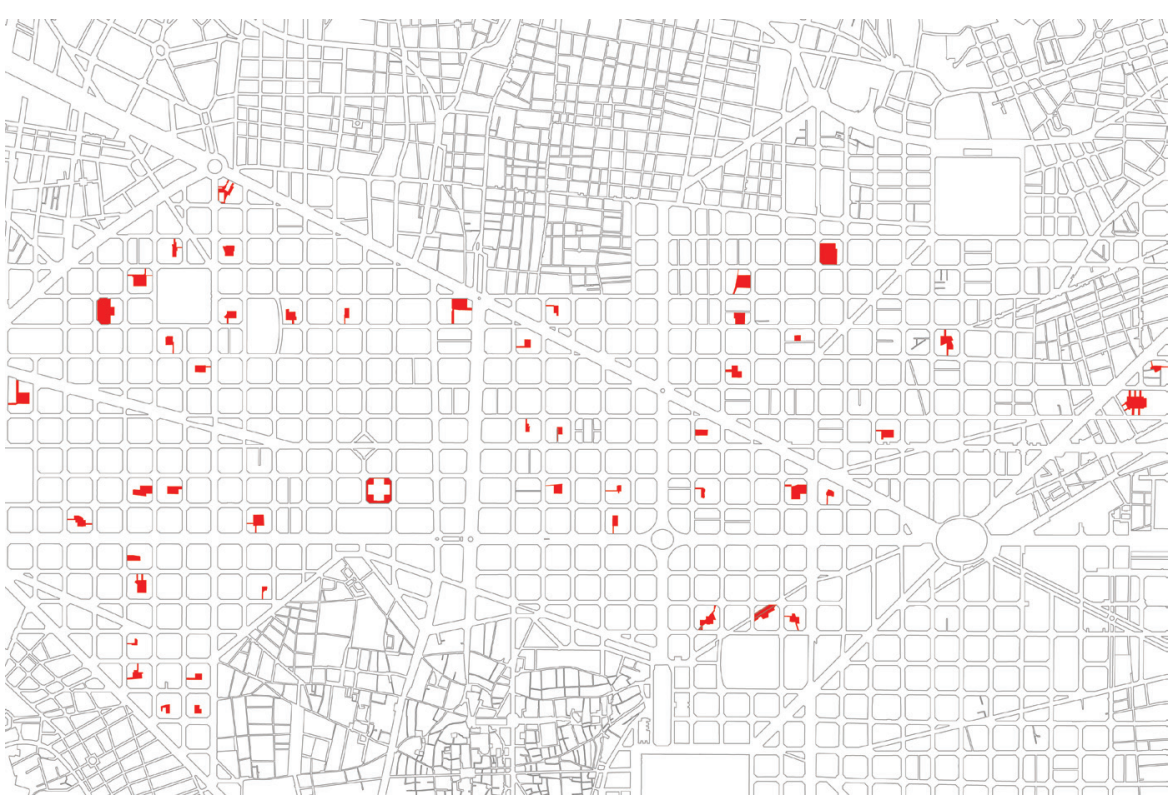
Fig. 1. Patios y
Dibujo propio.

más deseada por el Ayuntamiento y a ello va encaminada su política de mejora del Eixample, pero no es siempre posible porque son necesarias parcelas de gran tamaño.

El nivel de uso lo marcan principalemente los siguientes factores: proximidad al usuario, bien a su residencia, lugar de trabajo o equipamiento al que acude -colegio, biblioteca-; visibilidad desde la calle, accesibilidad; atractivo, interés; interacción o relación con otros patios próximos.

\section{Tanteos metodológicos para verificar el funcionamiento de los espacios} libres

Durante varios meses se realizó un trabajo de campo sobre la funcionalidad de los patios interiores de uso público. Consistió en el estudio cuantitativo y cualitativo de los usos en una muestra significativa de los distintos espacios interiores. Se escogieron patios que pudieran representar las diferentes tipologías, para poder extrapolar datos y obtener resultados globales. Se tuvieron en cuenta el número de accesos, la presencia de equipamientos en relación así como la proximidad a otros patios. En el estudio cuantitativo se contabilizó el número de personas que utilizaban el patio a lo largo del día, durante la semana y en horas punta. Estos 


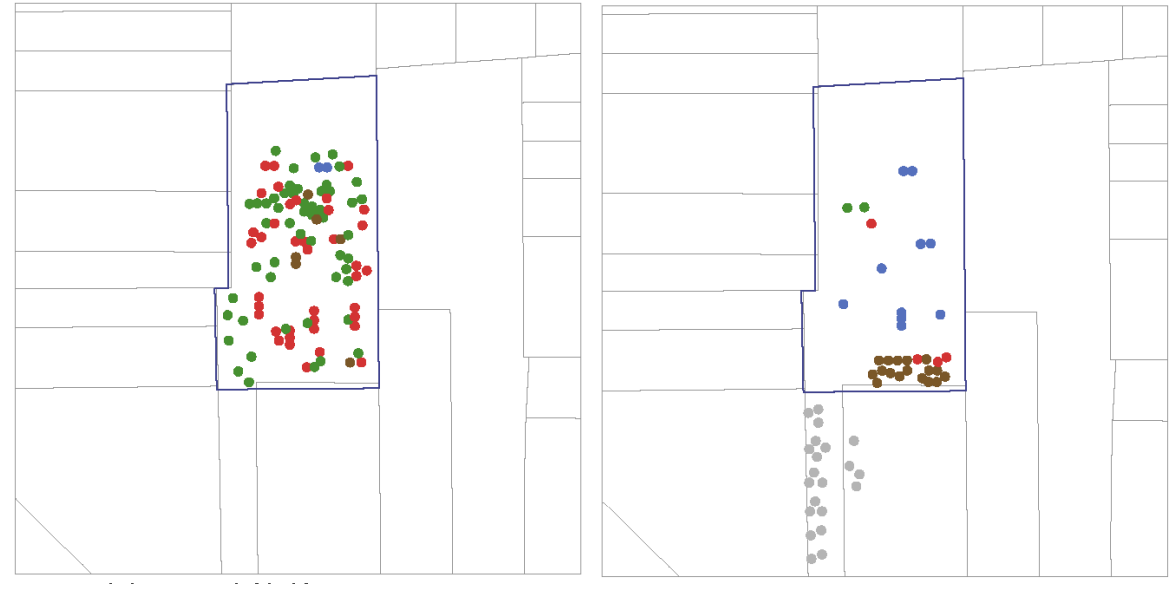

Fig. 2 y Fig 3. Esquemas de ocupación de los jardines de Joan Brossa, en dos momentos del mismo día, a las 1 th y a las
18h. En el primer caso gente los adultos se concentran en la zona de terrazas de un hotel y un restaurante, mientras que en el segundo caso son niños y sus cuidadores los que ocupan prácticamente todo el recinto, ya sea la zona de juegos como la
explanada y los bancos.

datos se mapificaron ${ }^{3}$ para estudiar la concentración por zonas (Fig. 2 y Fig. 3). Una segunda parte del estudio consistió en hacer encuestas de opinión y hábitos de uso a las personas que utilizaban el recinto. Esta información ayudó a complementar los estudios cuantitativos y a dar respuesta a muchas preguntas surgidas durante el trabajo de campo.

Los espacios liberados para uso público en los interiores de manzana no tienen un patrón común. Su tamaño y forma dependen de la manzana en la que se encuentran. Son diversos y por tanto disponen de una cierta flexibilidad a la hora de establecer los usos. Pero en general, en ellos se distinguen distintas zonas: de vestíbulo, la ajardinada, de juegos infantiles, la deportiva y la explanada. Cada una cumple una función: proporcionar el acceso, dotar de arbolado y frescor, facilitar entretenimiento y espacio de relación y esparcimiento.

Los usuarios mayoritarios son niños menores de 12 años y las personas que les acompañan, padres, cuidadoras o abuelos. Las actividades principales que se realizan están por tanto relacionadas con el juego: carreras, uso de balón, manipulación de tierra y agua, patinaje o bicicleta, aparte de la proporcionada por aparatos concretos como columpios, balancines, toboganes, castillos. Los adultos realizan una labor de vigilancia pero también de relación con otras personas: es un lugar de convivencia, de intercambio de conocimientos,

3 Se elaboraron unos esquemas donde se representaba la posición de los usuarios, diferenciando tipos, en momentos concretos del dia. Estos esquemas permite comparar de una manera más grááca la evolución del uso y el tipo de usuade ocupación por zonas y el tipo de usuario mayoritario, tanto por zonas como en global o respecto a otros patios. Para la mapificación se siguieron los trabajos de Bill Hillier dentro del Space Syntax laboratory, aunque él se centra más en espacios públicos de gran tamaño y en los flujos de personas y transportes. http://www.spacesyntax.com de encuentro intergeneracional. También hay personas que los usan para descansar, leer o por placer, si bien no son el grupo más numeroso.

La información obtenida mediante las encuestas en este ámbito ha sido de extraordinaria utilidad.

Se pudieron extraer resultados sobre la intensidad de uso, los hábitos de las visitas, la duración de las mismas, los gustos y necesidades, el grado de ocupación por zonas, las carencias, defectos y virtudes, así como el tipo de usuarios y las actividades realizadas.

Distinguiendo usos por zonas se puede decir que mayoritariamente la gente se concentra alrededor de los bancos, en lugares asoleados en primavera y sombreados en verano, y en proximidad a los juegos infantiles, que es donde se concentran los niños. Los recorridos dependen del número de accesos y del diseño. Más accesos favorecen zonas más transitadas. Los usuarios procuran hacer suyos los espacios pero el diseño les condiciona ya que está muy definida la actividad de cada zona. El espacio para la espontaneidad es a veces más limitado. Las explanadas son los lugares que permiten mayor improvisación y por ello son zonas de continuo movimiento de personas. Los accesos, cuando son en pasillo no se ocupan, son lugares de paso. Cuanto más amplio y más abierto es, permite mejor otra utilización. Las zonas ajardinadas tienen un uso limitado que en muchos casos se queda en el estético, y se cierran a paso para que no sena dañadas. En un espacio tan de mínimos la vegetación debería adaptarse en cierta manera al uso, es decir, si son espacios de mucho tránsito se deberían elegir especies resistentes, que puedan cumplir su función ambiental pero también estética e incluso aguantar el paso continuo, y muchas veces destructivo, de nińos. Los patios resultan ser paisajes urbanos de uso.

En cuanto a los tiempos de utilización las puntas horarias de uso ocurren a la tarde, sobre las $18 \mathrm{~h}$, coincidiendo con la salida de nińos del colegio. Uno de los mayores índices de ocupación detectado, personas por superficie, se midió en los jardines de Ermessenda de Carcassona y fue de $0,13 \mathrm{p} / \mathrm{m}^{2}$. Se contabilizaron hasta 131 personas en $1.028 \mathrm{~m}^{2}$. Otra información de interés obtenida se refiere a la duración de las estancias y las frecuencias de uso de los patios de manzana. En su mayoría son estancias medias de más de 30 minutos y que no superan las dos horas, según el día y las condiciones meteorológicas. Se frecuentan una vez al día y casi todos los días entre semana. En el fin de semana, cuando se dispone de más tiempo y horarios menos marcados, las personas encuestadas prefieren desplazarse a más distancia para disfrutar de lugares más adecuados a necesidades específicas: zonas más amplias, con mesas de picnic, canchas de deporte, espacios para correr o montar en bici. Buscan espacios de expansión, como la playa o los parques metropolitanos como Montjuic o Collserola. 
No obstante, los datos del estudio confirmaron que los ciudadanos acuden habitualmente al mismo espacio de recreo y que suelen recorrer distancias cortas, menos de $300 \mathrm{~m}$, para llegar a dicha zona. En el caso de los niños, menores de edad y acompañantes, suele ser una parada obligada que marca e recorrido diario del colegio a casa. Los patios se comportan así como prolongación del espacio de equipamiento, en este caso docente. Este hecho se hace todavía más patente cuando en la misma manzana existe un equipamiento con acceso compartido con el patio, como es el caso de guarderías, bibliotecas o centros de día. Los espacios libres interiores de manzana complementan, en efecto, a los recintos públicos (Fig. 4).

Asimismo, mediante el presente trabajo, se llegó también a la conclusión de que determinados factores influyen en el éxito del espacio público del interior de manzana. Las características del propio patio, la cantidad y tipo de accesos, el mobiliario urbano y otros elementos (bancos, juegos para nińos de edades variadas, aparatos para gente mayor), la calidad del espacio y su diseño, la iluminación, el arbolado y la vegetación, el atractivo o aquellas particularidades que lo hagan único, y también la capacidad de carga, es decir, cuánta gente pueda soportar sin perjudicar el uso adecuado, son factores internos o inherentes al propio espacio.

Por factores externos se entienden aquellos que existen independientemente de la presencia del patio, como sería la actividad en el entorno próximo, tanto laboral como comercial, o la que generan colegios y otro tipo de equipamientos. Otros factores externos a destacar serían la estacionalidad (la variación de las condiciones meteorológicas según las estaciones), el soleamiento, que depende a su vez del momento del año y de la altura de los edificios en el contorno del patio; la presencia de otros jardines próximos (que atraen a usuarios o los desvían a otras zonas), la organización de actividades por entidades como ProEixample ${ }^{4}$, y finalmente la publicidad que se haga de los patios para hacerlos más visibles en el barrio.

Un tercer factor mixto, que depende tanto del diseño del patio como de la actividad económica de su entorno puede ser la instalación de cafeterías con acceso desde el propio interior, que den servicio a los usuarios ${ }^{5}$.

En cuanto a su apreciación, destaca la valoración positiva de los ciudadanos respecto a los espacios interiores de manzana. Los encuentran seguros, próximos, bien mantenidos, bien dotados y agradables. El problema más repetido es la falta de espacios para niños mayores de 12 ańos, que requieren superficies más amplias para juegos más dinámicos, incompatibles generalmente en estos espacios relativamente pequeńos, enclaustrados y altamente ocupados. Otras críticas se dirigen a los materiales del pavimento demandando un ma-

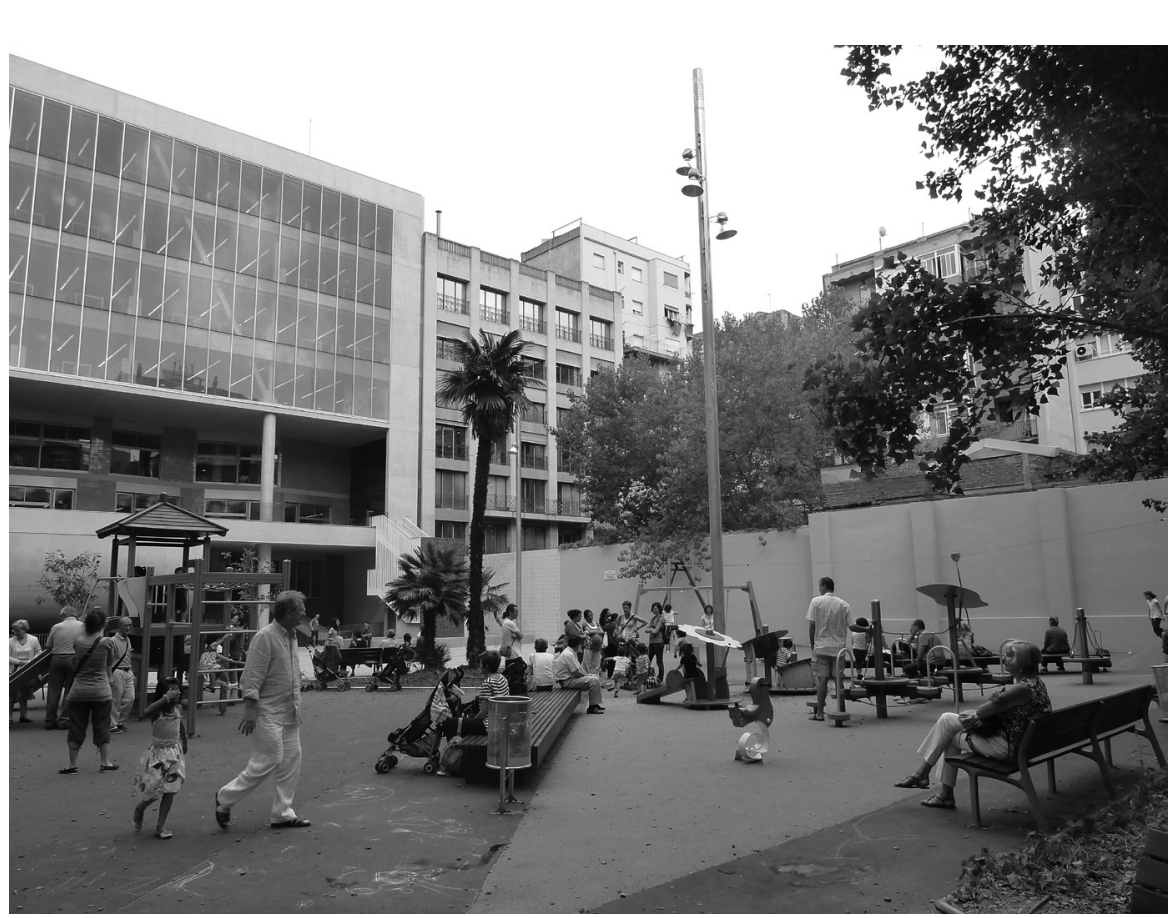

Fig. 4. En los jardines de Ermessenda de Carcassona, donde conviven patio y equipamiento multiusos (biblioteca, guarderí centro civico) la ocupación es de las más elevadas, incluso en fin de semana.

yor equilibrio entre materiales duros y blandos y más seguros, sin aristas que consideran peligrosas (Fig 5).

Curiosamente nadie opina sobre el arbolado y la vegetación, pese a que influyen notablemente en la percepción del espacio y la calidad del mismo Por ejemplo no aprecian o destacan el hecho de que los árboles frondosos contribuyen a cerrar el espacio creando un techo vegetal que favorece la intimidad y el silencio, además de la protección respecto de las vistas desde los edificios que los rodean. Ni tampoco sobre otras sensaciones que produce el verde, como corrector de la temperatura, de aromas o que permite apreciar el cambio de las estaciones. Es por ello que sería deseable insistir y divulgar estos servicios ambientales para aprender a valorarlos.

En una fase posterior se realizaron encuestas de opinión a personas que utilizaban espacios libres próximos a patios, como jardines o calles peatonalizadas, que ofrecen servicios de juegos infantiles y zonas arboladas. La intención era contrastar las diferencias de uso con respecto a los patios y de paso averiguar el tipo de usuarios que acudían. Se eligieron aquellos espacios próximos a algún patio, donde pudiera existir la opción de escoger entre uno y otro por 


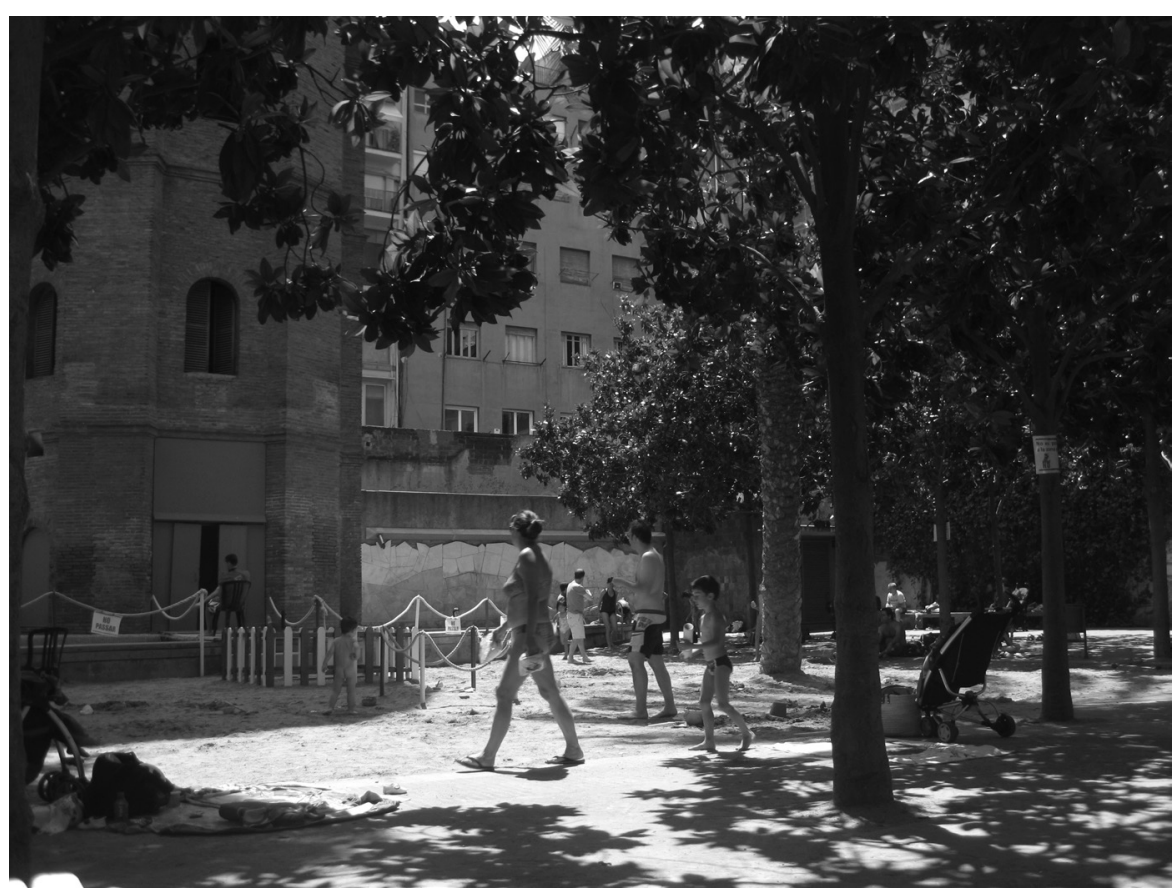

Fig. 5. El patio de la Torre de les Aigües destaca por su radical cambio de uso en invierno y verano. De julio a septiembre se convierte en una "playa" urbana desbordante de actividad, con piscina, arena para juegos, duchas y cambiadores, mientras que

la proximidad. Las conclusiones fueron bastante aclaratorias aunque no concluyentes: cada tipología de espacio libre atrae a tipos de usuarios según sus necesidades. Si hay opciones la gente escoge. Se detectaron tipos más variados de usuarios, no solo niños menores de 12 años y cuidadores sino también gente mayor sola o con amigos, que prefieren espacios más animados, con más actividad. En muchos casos no acuden a patios por desconocimiento, lo que demuestra una falta todavía de visibilidad de los patios desde el exterior. En general escogen los patios por su tranquilidad o porque son espacios seguros para los niños, sin tráfico.

Como conclusión cabe resaltar de nuevo el importante valor de los patios interiores públicos de manzana. Con su recuperación, y con otras actuaciones, se pretendía mejorar la calidad de vida del Eixample, y se ha conseguido. Son espacios pequeńos pero de enorme vitalidad, que permiten crear relaciones entre vecinos además de mejorar la calidad ambiental y estética de los interiores de manzana. Mejora la vida del residente pero también del visitante que acude, sea a descansar, a jugar con sus hijos o simplemente a disfrutar de un espacio protegido de coches y de la actividad de la calle.
Varias ideas se sugieren a partir del estudio de sus usos como la necesidad de una mayor visibilidad y conocimiento por parte de los vecinos y la búsqueda de un diseño para un uso más continuado y menos específico, que permita ampliar los tipos de usuarios y a diferentes horas del día o de la semana. Pero siempre con respeto hacia el vecindario, sin romper el frágil equilibrio entre el espacio público y la intimidad del espacio privado de las viviendas de la manzana.

A partir de la positiva relación que se ha observado entre equipamientos de barrio y patios interiores se abre la posibilidad de promover nuevas actividades que ampliaran esta relación fortaleciendo así el sentimiento comunitario y la participación ciudadana en esta escala inferior al barrio. Actividades que podrían ser por ejemplo, el intercambio de libros en las bibliotecas, actividades al aire libre de centros de mayores o guarderías, restauración o centros de reunión de barrio. Este hecho favorecería una mayor implicación y coordinación entre los departamentos municipales de los que dependen, como parques y jardines, educación o servicios sociales. La liberación del espacio interior de las manzanas posibilitaría además nuevos usos en las plantas bajas interiores, ya sea comercial, de oficinas o de servicio público, siempre que no se rompiera el frágil equilibrio entre intimidad y actividad.

Los patios interiores así como los espacios libres en general, como parte de los sistemas generales que son, forman parte de una red que se extiende por toda la ciudad y que debería potenciarse para mejorar aún más el uso y su correcto funcionamiento.

\section{Bibliografía}

AJUNTAMENT DE BARCELONA, 1983-1993. Anàlisi de l'Eixample. Modificació de l'ordenança per a la rehabilitació i millora, Direcció de Serveis de Planejament, Barcelona, 1993.

JACOBS, JANE, The Death and life of great American cities. Nueva Tork, Random House, 196

SPACESYNTAX, HILLIER, BILL, Proyecto de investigación para la Estación Victoria, Londres, 2007 consultado en web mayo 2011, http://www.spacesyntax.com/project/victoria-station/

Web de ProEixample: www.proeixample.cat 